\title{
Wilhelm Löffler (1887-1972) als Medizinhistoriker
}

\author{
Von Marie-Louise Portmann
}

Die medizinhistorischen Arbeiten Wilhelm Löfflers können nicht richtig gewürdigt und verstanden werden, wenn man nicht zuvor einige Streiflichter auf seine Persönlichkeit und seinen Werdegang wirft. Er wurde am 28.Juni 1887 in Basel als Sproß einer Kaufmannsfamilie geboren. Bereits während seiner Schulzeit in seiner Heimatstadt eignete er sich eine umfassende naturwissenschaftliche und allgemein humanistische Bildung an. Das Studium der Medizin begann er in Genf, um es in Basel und Wien fortzusetzen ${ }^{1}$. Schon als Student hatte er eine starke Neigung zur theoretischen Medizin, und es war Gustav von Bunge (1844-1920), Ordinarius für physiologische Chemie in Basel, der großen Einfluß auf Löffler ausübte. Bunge bestärkte ihn auch in seinem Hang zur Philosophie.

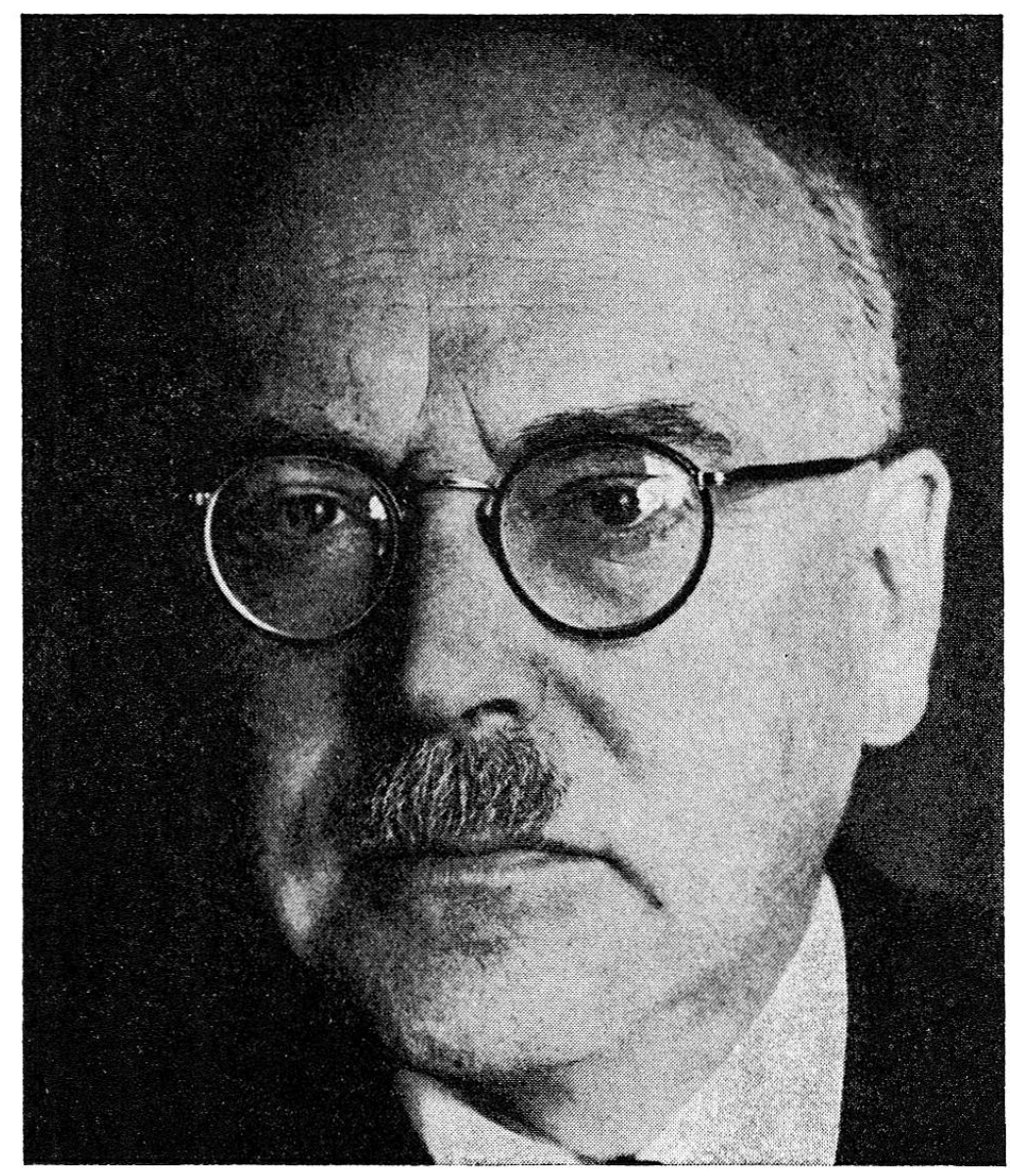

Prof.Dr.med. Wilhelm Löffler (1887-1972) 
Löffler erzählt: "Als er mich eines Tages dabei überraschte, wie ich, einen längeren Reaktionsverlauf abwartend, Kants Kritik der reinen Vernunft las, klappte ich, verlegen, mein Buch zu. Da meinte er mit seinem scharfen, baltischen Akzent: 'Lesen Sie weiter, lesen Sie nur Philosophie, bald genug werden Sie damit aufhören, aber es wird doch immer etwas davon an Ihnen haften bleiben.' ${ }^{2}$

1912 arbeitete Löffler seine Dissertation aus unter der Leitung Alfred Gigons (1883-1975), der sich gerade damals habilitierte. Nach seiner Promotion widmete sich Löffler weiter der Laboratoriumsarbeit. Zusammen mit dem Chemiker Markus Guggenheim (1885-1970), den Löffler als einen Mann von außergewöhnlicher biologischer Begabung bezeichnet, machte er Versuche über die Wirkungen und das Schicksal proteinogener, von Guggenheim später biogene genannter Amine im Tierkörper ${ }^{3}$ 1917 habilitierte sich Löffler in Basel für innere Medizin, und als Bunge im November 1920 starb, wurde der junge Dozent während zweier Semester mit der Vorlesung über physiologische Chemie betraut. Dabei wäre er, wie er sich launig ausdrückt, beinahe in diesem Fach hängengeblieben. Er sei aber schon damals durch die Klinik, das heißt durch den Kontakt mit dem lebendigen, leidenden Menschen, zu sehr verdorben gewesen, um sich definitiv für das theoretische Fach der physiologischen Chemie zu entscheiden ${ }^{4}$. 1921 erhielt dann Löffler einen Ruf nach Zürich, wo er die Leitung der Universitätspoliklinik übernahm und den Titel eines Extraordinarius erhielt.

Die große Bedeutung, welche Löffler zeit seines Lebens der Physiologie beimaß, kann aus einem Zitat ersehen werden, das ich seinem Vortrag «Die Beziehungen von Mensch zu Mensch» entnehme. Dieses Referat hielt er an der Jahrestagung der Schweizerischen Naturforschenden Gesellschaft in Aarau im Oktober 1960. Löffler behandelt sein Thema sehr stark unter historischen und philosophischen Aspekten und sagt dort: "Anthropomorph gesehen, ist die Physiologie als Lehre von den Funktionen in der belebten Welt als Königin der Naturwissenschaften bezeichnet worden. Auch die Geisteswissenschaften haben ihre Königin, die Geschichte. Über beide breitet die Philosophie ihren Baldachin aus. Von ihr aus geht der Blick ins Metaphysische. ${ }^{5}$

Doch müssen wir die Bedeutung Löfflers als Internist wenigstens kurz streifen. Als Vorsteher der Zürcher Universitätspoliklinik fand er zwei Syndrome, die seinen Namen tragen. Das erste, über das er 1932 eine Publikation veröffentlichte, sind die flüchtigen Lungeninfiltrate mit Bluteosinophilie, Lungenverschattungen, die sehr leicht mit Tuberkulose verwechselt werden können und die mit einer charakteristischen, ebenfalls flüchtigen Blutveränderung einhergehen. Sie werden oft, aber nicht immer, durch Wanderung von Spulwurmlarven durch die Lunge verursacht $^{6}$. Das zweite Syndrom, dessen Beschreibung in das Jahr 1936 zu 
datieren ist, betrifft die Endocarditis parietalis fibroplastica ? Sie besteht aus einer mit charakteristischen Blutveränderungen einhergehenden, diffusen Entzündung der Herzinnenwand mit hochgradiger Stauung besonders der Leber und der Milz.

1937 wurde Löffler nach der Emeritierung Otto Naegelis Ordinarius und Leiter der medizinischen Klinik der Universität Zürich. Als solcher wurde der Basler ein Nachfolger so bedeutender Mediziner wie Johann Lucas Schönlein (17931864), Carl Pfeufer (1806-1869), Hermann Lebert (1813-1878), Wilhelm Griesinger (1817-1868) und anderer. Zürich hat es verstanden, durch Berufung hervorragender Ärzte an seine medizinische Fakultät stets direkten Anteil an der Entwicklung der Heilkunde zu nehmen. Als dann der Regierungsrat des Kantons Zürich als Jubiläumsschrift zur 600-Jahr-Feier des Eintritts Zürichs in die Eidgenossenschaft sowie zur Eröffnung des neuen Kantonsspitals eine zweibändige Spitalgeschichte in Auftrag gab, lag es nahe, daß Löffler es übernahm, seine Vorgänger im Amt zu würdigen, was ihm denn auch nicht zu schwer fiel. Sein Beitrag in Band II, der 1951 erschien, umfaßt 228 Seiten und trägt den Titel «Die medizinische Klinik Zürich 1833-1950». In dieser Darstellung wurden die Persönlichkeiten sämtlicher Vorsteher seit der Gründung der Universität gemäß ihrer Herkunft, ihrem Werdegang, ihren Leistungen und ihren charakterlichen Eigenschaften vorgestellt. Daß die Würdigung der verschiedenen Klinikvorsteher etwas heterogen wurde, mag an der verschiedenartigen Quellenlage liegen. Denn nur bei der Darstellung Karl Ewald Hasses (1810-1902), welcher der Klinik von 1844-1852 vorstand und den Löffler als den wohl liebenswürdigsten unter seinen Vorgängern bezeichnet ${ }^{8}$, konnte er aus dessen persönlichen Lebenserinnerungen schöpfen, die in Leipzig 1902 unter dem Titel «Erinnerungen aus meinen Leben» erschienen waren. Als letzten Klinikvorsteher porträtiert Löffler sich selbst in einer ganz und gar unprätentiösen Darstellung.

Außer Bernhard Milt (1896-1956), der in Bd. I der Zürcher Spitalgeschichte eine Arbeit über die Geschichte des Zürcher Spitals schrieb, ist Löffler der einzige, der seinem Beitrag einen Anmerkungsapparat beigibt, in welchem er die von ihm erfaßten Fakten belegt. Damit weist sich Löfflers Arbeit als eine echte wissenschaftliche Leistung aus, wenngleich er nicht vollkommen konsequent die Quellen all seiner Lesefrüchte bekanntgibt. Seiner Darstellung stellt Löffler ein Motto voran, das er William Martins «Histoire de la Suisse» entnimmt. Es lautet: «Sans larges perspectives, les réalités du passé restent insaisissables.» ${ }^{9}$ Diese weiten Perspektiven hatte sich Löffler im wahrsten Sinne zu eigen gemacht. Dies soll ein Zitat aus seiner Einleitung illustrieren. Wie bereits erwähnt, bestand seine Aufgabe darin, die Entwicklung der Klinik von 1833 bis 1950 zu schildern. Er 
schreibt darüber: «Es ist überaus anziehend, der Entwicklung der Heilkunde in diesem Zeitabschnitt zu folgen, zu erkennen, wie einzelne Gesichtspunkte schon durchaus modern anmuten, wie aber andere gewissermaßen aus einer andern Welt zu kommen scheinen, wie Praxis und Theorie bald Hand in Hand gehen, bald in schroffem Gegensatz stehen, und vor allem, wie die Ärzte trotz ihrer beschränkten Hilfsmittel doch bedeutende Leistungen zu vollbringen vermochten. Nur wenn der Ausgangspunkt bekannt ist, kann der zurückgelegte Weg ermessen und können seine Mühsale richtig gewürdigt werden.»10

Am Anfang von Löfflers Darstellung steht die zentrale Gestalt von Johann Lucas Schönlein, dessen Ausstrahlung auf seine Schüler, von denen vier spätere Vorsteher der Zürcher Klinik wurden, sowie auf die gesamte Entwicklung der Medizin im deutschen Sprachgebiet Löffler meisterhaft zu schildern versteht. In einer graphischen Darstellung auf S. 77 gibt er einen Überblick über die Schüler Schönleins und Johannes Müllers (1801-1858) in ihren Abhängigkeiten und gegenseitigen Beziehungen. An die Spitze stellt er den Physiologen, Chirurgen und Augenarzt Philipp von Walther (1782-1849), der eine Zeitlang in Bamberg, woher Schönlein stammte, Vorsteher des Augen-Instituts war. Einer seiner Lehrsätze, der eben auf Schönlein Einfluß ausüben sollte, lautete: «Die Medicin kann wahre Fortschritte nur dadurch machen, daß die ganze Physik, Chemie und alle Naturwissenschaften auf sie angewendet und daß sie auf die gegenwärtig erstiegene Höhe derselben gestellt und mit ihren glänzenden Fortschritten in Übereinstimmung gesetzt werde. ${ }^{11}$ Diese Forderung stand im Gegensatz zu der von Friedrich Wilhelm Joseph von Schelling begründeten Naturphilosphie, in deren Anschauungen Schönlein in seinen Anfängen noch wurzelte. Löffler, durch dessen Darstellung sich die philosophischen Grundlagen wie ein roter Faden ziehen, hebt hervor, daß die Nötigung, sich mit der absterbenden Naturphilosophie zu beschäftigen, und die Suche nach einer besseren Philosophie dazu führten, daß die Mediziner die allgemeinen Fragen nicht aus den Augen verloren ${ }^{12}$. Schönlein kam mit seiner Methode und seiner Schule, die er die naturhistorische nannte, den obenerwähnten Forderungen Walthers vollkommen nach. Er lehrte seine Schüler ein ärztliches Verhalten am Krankenbett, das den Namen Beobachtung wirklich verdient. Dazu gehörte die Übernahme der Perkussion und Auskultation, die durch Schönlein und seine Schüler im deutschen Sprachgebiet verbreitet wurden. Löfflers Hauptverdienst ist es, die Leistungen Schönleins während seiner Zürcher Zeit gebührend gewürdigt zu haben. Der Umfang von Schönleins literarischem Gesamtwerk war zwar klein: zwei Arbeiten auf drei Seiten. Eine dieser beiden Schriften zeichnet sich jedoch dadurch aus, daß sie die Entdeckung des ersten exogenen pflanzlichen Krankheitserregers enthält ${ }^{13}$. 
Als Schönlein 1839 nach Berlin zog, wurde Carl Pfeufer sein Nachfolger, der ebenfalls aus Bamberg stammte und der in Würzburg eine Zeitlang Assistent Schönleins gewesen war. Löffler hebt die Bedeutung von Pfeufers Freundschaft mit dem Anatomen und Histologen Jacob Henle (1809-1885) hervor, der ein Schüler Johannes Müllers war und zu gleicher Zeit wie Pfeufer in Zürich eine Professur bekleidete. Die beiden Freunde waren Vertreter der sogenannten rationellen Medizin, die sich auf Vernunft und Erfahrung stützte und auf wissenschaftlicher Erkenntnis beruhte. Um ihr Programm zu verbreiten, gaben sie die «Zeitschrift für rationelle Medicin» heraus, deren erste zwei Bände 1844 in Zürich gedruckt wurden ${ }^{14}$.

Im selben Jahr verließen die beiden Dioskuren, wie Löffler sie nennt, die Zürcher Universität, um einem Ruf nach Heidelberg zu folgen. Karl Ewald Hasse, der von 1844 bis 1852 der Klinik vorstand, schildert Löffler als einen Arzt, der zwar indirekt unter Schönleins Einfluß stand, ihm aber bereits kritisch wertend gegenübertrat. Hermann Lebert, Klinikvorsteher 1853-1859, hatte in Zürich bei Schönlein studiert. Wilhelm Griesinger, den Löffler als den letzten Schönlein-Schüler würdigt, war zusammen mit seinem Lehrer und Freund Carl August Wunderlich (1815-1877) der Vertreter einer neuen medizinischen Richtung, der physiologischen Heilkunde, die sich gegen die Herrschaft der naturhistorischen Schule richtete, deren Begründer Schönlein war ${ }^{15}$. Die neue Richtung stützte sich auf die Erkenntnisse der Morphologie, Anatomie und Histologie und versuchte, die einzelnen Lebensvorgänge mit physikalischen und chemischen Methoden zu analysieren, in ihrem Mechanismus aufzuklären und durch Synthese der Einzelfunktionen die Gesamtfunktionen und die Verhaltensweisen des Organismus zu verstehen. Die Begründer der physiologischen oder physikalischen Medizin bekämpften die rationelle Medizin Pfeufers.

Das Jahr des Amtsantritts von Anton Biermer (1827-1892), der 1865 nach Zürich kam, schildert Löffler als einen Wendepunkt für Naturwissenschaft und Medizin, indem bedeutende Veröffentlichungen in dieses Jahr fallen. Er zählt dazu die «Geschichte des Materialismus» von Friedrich Albert Lange (18281875). Löffler ist so richtig in seinem Element, wenn er auf die neuen theoretischen Grundlagen der Medizin in der zweiten Hälfte des 19.Jahrhunderts zu sprechen kommt, wobei er bedeutende Autoren durch prägnante Zitate zu Worte kommen läßt. Seine Darstellung stützt sich sowohl auf die Vertreter des Materialismus als auch auf deren Gegner. Er vertritt jedoch die Ansicht, daß die materialistische Auffassung, die aus der konkreten Naturbetrachtung neu emporgewachsen war, sich eher fördernd auf die Krankheitsbekämpfung und die Gesundheitsförderung für die Gesamtheit ausgewirkt habe ${ }^{16}$. Die Klinikvorsteher 
Anton Biermer, Gustav Huguenin (1841-1920) und Hermann Eichhorst (18491921) nenne ich nur. Löffler würdigt sie im zweiten Teil seiner Darstellung, der die Jahre 1865-1950 umfaßt. Dieser zweite Teil ist bei weitem weniger umfangreich als der erste Teil, der die Jahre 1833-1865 umspannt.

Die Schilderung seines unmittelbaren Amtsvorgängers Otto Naegeli (18711938), den er persönlich kannte, geschah aus eigenem Erleben. Es gelang ihm vor allem sehr gut, die menschlichen Seiten dieses Arztes ins Licht zu rücken. Das abschließende Selbstporträt Löfflers wurde schon erwähnt.

Dieser Beitrag zur Zürcher Spitalgeschichte ist nicht die einzige medizinhistorische Leistung des Zürcher Klinikers. Als Internist hatte sich Löffler sehr intensiv mit der Tuberkulose zu befassen, und er erlebte selbst die gewaltigen Fortschritte, die in der Behandlung dieser Krankheit bis zur Einführung des Streptomycins und der aus den Laboratorien der chemischen Industrie hervorgegangenen Antituberkulosa erzielt wurden. Sein großes Engagement auf diesem Gebiet und seine profunden Kenntnisse bewogen ihn dann, eine Geschichte der Tuberkulose zu verfassen, die 1958 in dem fünfbändigen «Handbuch der Tuberkulose» erschien, das von J.Hein, H. Kleinschmidt und E. Uehlinger im Georg Thieme Verlag herausgegeben wurde ${ }^{17}$. Im Gegensatz zu etwa gleichzeitig erschienenen Veröffentlichungen über dasselbe Thema (Richard Bochalli 1940, 1958, Selman A. Waksman 1964) ${ }^{18}$ zeichnet sich Löfflers Darstellung durch große Übersichtlichkeit, sorgfältig ausgewähltes Bildmaterial und vor allem durch einen exakten Anmerkungsapparat aus, der in den Darstellungen der anderen Autoren fehlt.

Löffler war sich bewußt, daß er als einer der letzten die Tuberkulose so schildern konnte, wie sie sich früheren Generationen darbot. Er schreibt: «Die Generation der heute alten Ärzte ist vielleicht die letzte, die die Volksseuche noch in ihrer ganzen ungeheuren Wucht miterlebt hat, die darum weiß, welch großer Anstrengungen und umfassender Organisationen es bedurfte, um die Krankheit einzudämmen, historisch gesehen, späte Anstrengungen. Jahrtausendelang hatte das Dogma der Unheilbarkeit lähmend gewirkt. »19

Einleitend gibt Löffler die wichtigsten Marksteine der Entwicklung wieder in Form einer chronologischen Tabelle. Den Anfang bildet Auenbruggers Erfindung der Perkussion (1761), und an den Schluß stellt er die Einführung des Streptomycins in die Tuberkulosetherapie durch Selman Abraham Waksman (18881973) und die Herstellung des Isonicotinylhydrazins (Rimifon) durch die chemische Industrie ${ }^{20}$.

Doch Löfflers historische Forschung geht viel weiter zurück bis ins Dunkel der Vorzeit. Im Kapitel über Prähistorie und Altertum spricht er über die ältesten bekanntgewordenen Zeugnisse tuberkulösen Geschehens, die in Knochenfunden 
von etwa 5000 v. Chr. an vorliegen. Auf Phthisen bei den Griechen und Römern geht Löffler ausführlich ein, während er die nachgalenische Periode nur summarisch behandelt. Im Kapitel über die moderne Zeit, die mit der Einführung der Perkussion und Auskultation beginnt, kommt Löfflers weiter Horizont recht eigentlich zur Geltung ${ }^{21}$. Im Abschnitt über die Heilstättenbewegung, der einen Einblick gibt in die strenge Disziplin, welche die Patienten zu befolgen hatten, konnte Löffler aus eigener Erfahrung sprechen, war er doch 1909 als Volontär an der Basler Heilstätte in Davos tätig gewesen, die damals von Dr. E. Nienhaus militärisch straff geleitet wurde. Löffler erblickt in der Liegekur, die zweifelsohne Erfolge zeitigte, einen magischen Gehalt, der auf der Intuition und der Energie einiger weniger beruhte, die sie einen Weg finden ließen, auf dem die Phthisiotherapie fast ein Jahrhundert lang fortschreiten konnte. Nach Löfflers Auffassung ist die Liegekur eine intuitive, künstlerische Schöpfung, die unter den Verfahren der Heilkunde eine Sonderstellung einnimmt ${ }^{22}$.

Ausführlich geht Löffler auf den Beweis der Übertragbarkeit der Tuberkulose ein, der 1865-1868 von Jean-Antoine Villemin geliefert wurde, sowie auf die Lehre von der Kontagiosität, die schon jahrhundertelang bei den Ärzten mehr oder weniger Anklang gefunden hatte ${ }^{23}$. Die Entdeckung des Tuberkelbazillus 1882 durch Robert Koch leitet ein neues Kapitel ein, das in dem mit «Tuberkulinsturm » bezeichneten Abschnitt seine Fortsetzung findet. Koch empfahl nämlich das Tuberkulin als Heilmittel, während Virchow vor dessen kritikloser Anwendung warnte ${ }^{24}$. Der Kochsche Grundversuch gibt den Stoff für einen neuen Abschnitt. Dieser Versuch zeigt, daß bei einem tuberkulös infizierten Tier eine zweite Infektion nicht mehr angeht ${ }^{25}$. Zwischen diesen Kapiteln finden sich immer wieder Fortsetzungen der Chronologie, d.h. Zusammenstellungen wichtiger Daten aus der Geschichte der Tuberkulose in lockerer Folge. Einen weiteren Schwerpunkt legt Löffler auf die Entdeckung der Primoinfektion, die auf Georges Küss zurückgeht, der das Gesamtbild der postnatalen aerogenen, tuberkulösen ersten Ansteckung beschreibt (1898) ${ }^{26}$. Der Stadieneinteilung durch K. E. Ranke ist ein weiteres Kapitel gewidmet. Löffler findet bei diesem Autor die ersten heuristisch wertvollen Klassifikationen tuberkulösen Geschehens (1916) ${ }^{27}$.

Die am Anfang des 20. Jahrhunderts von vielen Autoren vertretene, später von den Verfechtern der Infiltrattuberkulose bekämpfte Lehre vom Spitzenbeginn der Krankheit gibt Löffler Gelegenheit, rückblickend seine vermittelnde Stellungnahme zur Geltung zu bringen ${ }^{28}$. Interessant sind die Paradoxa in der Entwicklung der Tuberkuloselehre, die Löffler in einem besonderen Abschnitt aufzeigt. Er legt dar, wie nicht selten tiefgreifende Irrtümer fördernd, wissenschaftliche Erkenntnisse dagegen hemmend auf die Maßnahmen zur Bekämpfung der Krank- 
heit eingewirkt haben ${ }^{29}$. Auch die Geschichte der Bronchoskopie und Bronchologie wird berücksichtigt ${ }^{30}$. Dann greift der Autor wiederum weit zurück, um die historische Bedeutung der Skrofulose darzulegen, von der man glaubte, daß sie durch die Berührung des Königs mit der Hand geheilt werden könne ${ }^{31}$. Schließlich gibt Löffler einen Rückblick auf die Therapie und faßt die gebräuchlichsten Pharmaka zusammen. Die Therapieformen sowie die Geschichte der chirurgischen Behandlung der Lungenkrankheiten werden wiederum in je einer Tabelle zusammengefaßt $\mathrm{t}^{32}$.

Den Schluß und die Krönung der ganzen Darstellung bildet das Kapitel über die antibiotische Periode. Löffler erzählt, wie er den ersten mit Streptomycin geheilten Patienten seinem Kollegen S.A. Waksman im Sommer 1947 vorstellen konnte. Alle, die um das Krankenbett standen, hätten gefühlt «die alte Zeit ist um $» .^{33}$

Auch Waksman berichtet in seiner Darstellung in einem Kapitel «A visit to the Zurich state hospital» über seinen Besuch bei Löffler, dessen fortschrittliche Therapie ihn beeindruckte. Waksman schreibt: "Climaxing other impressions gained from other types of patients and in other countries, what I saw here stimulated my own morale. The life-saving properties of streptomycin were a reality after all! It is to be recalled that this was in 1947, when the only detailed studies of the effect of streptomycin in experimental and clinical tuberculosis were still being made largely in the United States. The British were still skeptical. The French had had little experience. The northern countries were still to be heard from. Russia had little to go by. I came away from that hospital more cheerful than I had been for some time." 34

Obwohl inzwischen auch der Pariser Medizinhistoriker Charles Coury eine mit einer umfangreichen Bibliographie versehene Monographie über die Geschichte der Tuberkulose verfaßt hat, die 1972 erschienen ist und auch die neueren Forschungen miteinbezieht ${ }^{35}$, ist Löfflers Darstellung damit keineswegs überholt. Durch ihre originellen Gedankengänge, die Darbietung vieler Fakten und ihre übersichtliche Gliederung ist sie auch weiterhin aktuell.

Löfflers Vortrag «Die Beziehungen von Mensch zu Mensch» (1960), der schon oben besprochen wurde, enthält ebenfalls medizinhistorische Aspekte. Dabei weiß sich der Verfasser geistesverwandt mit seinem Landsmann Jacob Burckhardt, dessen kulturkritische «Weltgeschichtliche Betrachtungen» er treffend zitiert $^{36}$.

Auf eine letzte medizingeschichtliche Arbeit Löfflers muß noch hingewiesen werden. Es ist dies der Vortrag «Zur Entwicklung der Beziehungen zwischen Chemie und Nosologie ${ }^{37}$. Der bereits erwähnte ehemalige Mitarbeiter Löfflers, 
Markus Guggenheim, hatte ebenfalls starke medizinhistorische Interessen. Dieser Biochemiker errichtete eine Stiftung, die es der Schweizerischen Gesellschaft für Geschichte der Medizin und der Naturwissenschaften unter anderem ermöglicht, alljährlich an ihrer Jahrestagung einen Vortrag halten zu lassen und dafür ein besonderes Honorar zu entrichten. Im September 1968 hielt Wilhelm Löffler in Einsiedeln in Anwesenheit Guggenheims den von diesem gestifteten Vortrag über das oben genannte Thema. Dabei erläuterte Löffler einen die beiden Forscher gemeinsam interessierenden Gegenstand, nämlich die Bedeutung der chemischen Forschung für die Medizin. Der Autor konnte dabei aus der reichen Erfahrung eines langen Gelehrtenlebens schöpfen. Damit schloß sich der Kreis für die beiden Gelehrten Löffler und Guggenheim. Der letztere starb im Jahre 1970 im Alter von über 85 Jahren. Löffler folgte ihm zwei Jahre später, am 25. November 1972, ebenfalls im 86. Lebensjahr. Der Zürcher Kliniker hat mit seiner medizinhistorischen Forschung wichtige Bausteine zur Medizingeschichte der Schweiz beigesteuert.

\section{Anmerkungen}

${ }^{1}$ Kartagener, Manes, Wilhelm Löffler zum 70. Geburtstag. Neue Zürcher Zeitung, 28. Juni 1957.

2 Zürcher Spitalgeschichte, Zürich 1951, Bd. II, S. 208.

3 Ebenda S. 209.

4 Ebenda S. 210.

${ }^{5}$ Löffler, Wilhelm, Die Beziehungen von Mensch zu Mensch. Verhandlungen der Schweizerischen Naturforschenden Gesellschaft, 140. Versammlung im Kanton Aargau 1960, S.59.

${ }^{6}$ Löffler, Wilhelm, Zur Differential-Diagnose der Lungeninfiltrierungen. I. Frühinfiltrate unter besonderer Berücksichtigung der Rückbildungszeiten. Beitr. Klin. Tbk. 79 (1932), S. $338-368,566$.

Löffler, Wilhelm, Zur Differentialdiagnose der Lungeninfiltrierungen. II. Über flüchtige Succedan-Infiltrate (mit Eosinophilie). Beitr. Klin. Tbk. 79 (1932), S. 368 ff.

${ }^{7}$ Löffler, Wilhelm, Endocarditis parietalis fibroplastica mit Bluteosinophilie. Ein eigenartiges Krankheitsbild. Schweizerische medizinische Wochenschrift 66 (1936), S. 817-820.

${ }^{8}$ Zürcher Spitalgeschichte, a.a.0. S.108.

${ }^{9}$ Ebenda S. 1.

10 Ebenda.

11 Ebenda S. 16.

12 Ebenda S. 22.

13 Ebenda S. 24.

14 Ebenda S. 102 f.

15 Ebenda S. 148.

16 Ebenda S. 163. 
17 Weitere Veröffentlichungen Löfflers zu demselben Thema sind die folgenden:

Chronologie der Erkenntnisse über die Tuberkulose. Handbuch der Inneren Medizin; Atmungsorgane, Bd. 3, Springer, Berlin 1956, S. 447-463.

Episoden aus der Geschichte der Aetiologie der Lungentuberkulose. Dermatologica (Basel) 115 (1957), S. 304-309.

Tuberkulose und Tuberkulosebekämpfung im Wandel der Zeiten. Schweizerische medizinische Wochenschrift 100 (1970), S. 1790-1802.

${ }^{18}$ Bochalli, Richard, Die Geschichte der Schwindsucht. Praktische Tuberkulose-Bücherei. Beihefte des Deutschen Tuberkulose-Blattes, 24.Heft, Georg Thieme Verlag, Leipzig 1940.

Bochalli, Richard, Die Entwicklung der Tuberkuloseforschung in der Zeit von 1878 bis 1958. Tuberkulose-Bücherei. Georg Thieme Verlag, Stuttgart 1958.

Waksman, Selman Abraham, The Conquest of Tuberculosis, University of California Press, Berkeley and Los Angeles 1964.

${ }^{19}$ Löffler, Wilhelm, Geschichte der Tuberkulose. In: Handbuch der Tuberkulose, Bd. 1, 1958, S. 2. (Für den Hinweis danke ich Erwin H.Ackerknecht.)

20 Ebenda S. 1-3.

${ }^{21}$ Ebenda S. 24ff.

22 Ebenda S. 37-47.

${ }^{23}$ Ebenda S. 47-54.

24 Ebenda S. 58-67.

${ }^{25}$ Ebenda S. 67.

${ }^{26}$ Ebenda S. 74-77.

27 Ebenda S. 77-79.

28 Ebenda S. 81-83.

${ }^{29}$ Ebenda S. $88 \mathrm{f}$.

30 Ebenda S. $87 \mathrm{f}$.

31 Ebenda S. 89-96.

32 Ebenda S. 96-105.

33 Ebenda S. 106.

${ }^{34}$ Waksman, Selman A., a. a. O. S. 158-160.

${ }^{35}$ Coury, Charles, La tuberculose au cours des âges, Lepetit S.A., Suresnes 1972.

${ }^{36}$ Löffler, Wilhelm, Die Beziehungen von Mensch zu Mensch, a.a. O. S. 59.

${ }^{37}$ Gesnerus 26 (1969), Heft 1/2, S. 1-25. 


\section{Summary}

Wilhelm Löffler, director of the University clinic in Zurich from 1937 to 1957, was a very versatile man who was also outstanding in the history of medicine. When the new state hospital was opened in 1951, the governmental council edited a history of the hospital in Zurich in two volumes to which Löffler contributed a historical appreciation of his predecessors as heads of the clinic from 1833 to 1937. He also added an autobiographical survey on his own person.

Löffler who was a famous clinician after whom two syndromes are named was also very up-to-date in the therapy of tuberculosis. After Selman Abraham Waksman (1888-1973) had introduced streptomycin into tuberculosis therapy in 1944, Löfler was one of the first who applied the new medicament in his clinic. He was aware of the beginning of a new era, and as one of the last physicians who had been able to become acquainted with the disastrous effects of the disease, he untertook the task of writing a history of tuberculosis which was published in the "Handbuch der Tuberkulose", edited by J.Hein, H. Kleinschmidt and E. Uehlinger in 1958. Löffler's survey is very lucid and it is commented by exact annotations. The text which has fine illustrations climaxes in the description of the meeting of Waksman and Löffler in Zurich in 1947 when the latter presented to his guest the patients whom he had cured by streptomycin. Waksman too remembers his visit in Zurich in his book "The Conquest of Tuberculosis" (1964).

Dr. Marie-Louise Portmann

Medizinhistorische Bibliothek

Schönbeinstraße 40

4056 Basel 